Sādhanā Vol. 36, Part 6, December 2011, pp. 977-994. (C) Indian Academy of Sciences

\title{
A general approach for optimal kinematic design of 6-DOF parallel manipulators
}

\author{
VIVEK KUMAR MEHTA and BHASKAR DASGUPTA* \\ Department of Mechanical Engineering, Indian Institute of Technology, \\ Kanpur 208 016, India \\ e-mail: dasgupta@iitk.ac.in
}

MS received 4 June 2010; revised 1 June 2011; accepted 19 August 2011

\begin{abstract}
Optimal kinematic design of parallel manipulators is a challenging problem. In this work, an attempt has been made to present a generalized approach of kinematic design for a 6-legged parallel manipulator, by considering only the minimally required design parameters. The same approach has been used to design a 7-legged redundant parallel manipulator. Two ways of introducing redundancy into the parallel manipulator have been demonstrated and comparison between them has been presented.
\end{abstract}

Keywords. Parallel manipulator; isotropy; kinematic design; force redundancy.

\section{Introduction}

The field of robotics has been greatly influenced by biological systems. Most of the industrial robots resemble the upper part of human arm, having several links connected serially. Such kind of robots or manipulators are said to possess serial structure, and hence, called serial manipulators or open-chain manipulators. These manipulators enjoy the advantage of having large and dexterous workspace. Thus, in applications where a large region in space needs to be reached, serial manipulators are used. But, due to their open-chain structure, they have some disadvantages, too. The main drawbacks are poor accuracy due to accumulation of link position errors, less stiffness and low load carrying capacity. To overcome these drawbacks, researchers have proposed manipulators with closed-chain structure, known as parallel manipulators.

A parallel manipulator is a closed-chain mechanism, generally consisting of a moving platform $(M P)$ and a fixed base $(F B)$ connected by several independently actuated serial kinematic chains or linkages (called legs). Due to their closed-chain structure, they have higher stiffness, high load carrying capacity and precise positioning capabilities. But, since there are several legs that apply a number of constraints on the motion of $M P$ with respect to $F B$, their workspace is much smaller in comparison to the serial manipulators. Besides, they suffer a severe disadvantage of singularity within the workspace. Gosselin \& Angeles (1990) present an analysis of the different kinds of singularities encountered in closed-loop chains.

${ }^{*}$ For correspondence 
The best known example of a parallel manipulator is the classical Gough-Stewart platform, as depicted in figure 1. It consists of $M P$ connected to $F B$ through six legs. Each leg consists of a prismatic actuator and two passive joints. The prismatic actuator allows the control of length of the leg. The joint at the $M P$ is a spherical joint and the joint at the $F B$ may be a spherical or universal joint. The motion of the $M P$ can be controlled in full six degrees of freedom (dof). It has been used as a motion base in flight simulators since 1960's Stewart (1965). The moving platform is often referred to as end-effector, in particular for machine tool application where a tool is rigidly attached to the $M P$. For detailed background on parallel manipulators, Merlet (2000) or Dasgupta \& Mruthyunjaya (2000) may be consulted.

Although it has been demonstrated in various applications, such as vehicle simulators, space manipulators, medical manipulators, welfare supporting machines, etc. that the parallel manipulator offers good performance, it still lags behind in certain potential application areas (e.g., machine tool application) where this type of structure is not yet amply utilized. One of the important reasons for this is the lack of computational tools that can efficiently design the parallel manipulators. This is an important issue as the performance of these manipulators is highly dependent on their dimensioning. This is a complex problem involving many parameters. Several criteria have been used in the past for designing parallel manipulators.

One of the important criteria in the optimal kinematic design is the ability of the manipulator to achieve an isotropic or near-isotropic configuration. In an isotropic configuration, the sensitivity of a manipulator in both velocity and torque measurement is at a minimum. It has been recognized that the condition number of Jacobian or some other equivalent metric is the most suitable index for evaluating the performance of the manipulator (Salisbury \& Craig 1982; Goselin \& Angeles 1991). The concept of isotropy is well accepted as a design criteria for obtaining optimal design (Dwarakanath et al 2001; Zanganeh \& Angeles 1997; Ma \& Angeles 1991; Gosselin \& Angeles 1988; Chablat et al 1998; Klein \& Blaho 1987; Huang et al 2004). Some of the authors (Fattah \& Hasan Ghasemi 2002; Fassi et al 2005) determine the isotropic conditions for the Jacobian matrix in a purely symbolic manner. Fattah \& Hasan Ghasemi (2002) have found these conditions for the special case of the translational motion of the platform and have concluded that even for this case it is not possible to design an isotropic parallel manipulator having symmetrical structure. Jafari \& McInroy (2003) have characterized and studied the properties of Orthogonal Gough-Stewart platform (OGSP's) over a small workspace. Further, they have used this characterization to design OGSP's that will achieve a desired hyper-ellipsoid of velocities.

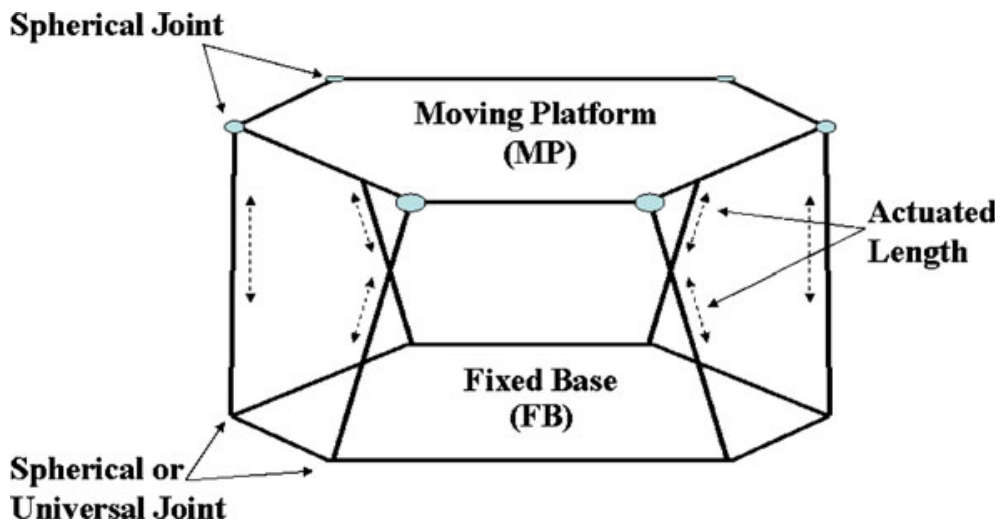

Figure 1. Classical Gough-Stewart platform. 
Apart from using isotropy as the design measure, some authors have also studied the design of parallel manipulators on the basis of dynamic measures. Ma \& Angeles $(1990,1993)$ introduced a measure of dynamic performance of manipulator, based on the concept of dynamic isotropy. Salcudean et al (1994) studied the effect of radii of platforms of the Stewart platform on the articular force necessary to obtain a given acceleration of the platform. Hao \& Merlet (2005) propose a multi-criteria optimal design of parallel manipulator based on interval analysis. Hay \& Snyman (2003) have studied the problem of determining a two or three dof manipulator design so that its workspace corresponds to a prescribed workspace using two different strategies. Some of the authors have also suggested few modifications on the existing design, so as to improve the performance of the existing manipulators in terms of increase in workspace (Miller 2002) or enhanced rotational capabilities (Liu et al 2005). Merlet \& Daney (2005) have presented the dimensional synthesis of parallel manipulators with a given positional accuracy over a specific workspace. In a deep analysis of the concepts of manipulability, isotropy and condition number for parallel robots as accuracy index, Merlet (2006) shows that, in spite of the utility of the condition number as a single scalar measure of the pose quality, it should not be expected to yield a one-to-one correspondence with inaccuracy, since the involved mapping is nonlinear. Stock \& Miller (2003) proposed a new performance index, space utilization for designing spatial parallel manipulator.

Several prototypes based on the Stewart platform have been built for machine tool applications. These prototypes have only six legs as used in the traditional design which is known to be handicapped by singularity within the workspace. It has been shown (see Dasgupta \& Mruthyunjaya 1998) that addition of an extra $\operatorname{leg}^{1}$ results in a tremendous enhancement in the dexterity with very little loss of workspace. Wang \& Gosselin (2004) address the singularity analysis and design of kinematically redundant parallel manipulators. The main idea in the design is the addition of one redundant degree of freedom in one of the kinematic chains of the nonredundant manipulator, but the addition of an extra degree of freedom in one of the kinematic chain destroys in-parallel characteristic of parallel manipulator.

On the basis of the literature related to design of parallel manipulators, it is apparent that most of the work has been done for optimizing the special cases of classical Stewart platform manipulator. Little work has been reported where design of the general case considers all the kinematic design parameters. Besides, very few papers discuss the problem of designing the redundant manipulators. As an added motivation, the quest for a design procedure for optimal design of redundant parallel manipulators would consolidate design procedures for future needs. In the present work, an attempt is made in that direction, wherein the proposed method attempts to achieve practically feasible designs. The method incorporates all the kinematic design parameters that are minimally required to define the geometry of parallel manipulators.

In the next section, the design method, optimization criteria and basic formulation are discussed in detail. Numerical results are presented in section 3 and, finally, the last section summarizes the present work.

\section{Formulation of optimal kinematic synthesis}

This section first deals with the conventionally used kinematic design methodologies as a preamble to the new method suggested in this work. Starting with a brief introduction to the parameters that affect the design procedure, the design objectives are established subsequently.

\footnotetext{
${ }^{1}$ Unlike redundancy in serial manipulators, here the addition of an extra leg does not increase the $d o f$.
} 


\subsection{Design parameters}

Kinematic design is an important aspect for the practical conceptualisation of a manipulator. This is so because the performance is largely dictated by the structure derived through the kinematic design procedure. This is the basic step of the entire design loop influencing other factors down the line. The optimal kinematic design problem involves determination of kinematic parameters such as link lengths, joint positions, etc. in such a manner that the resulting design fulfils certain requirements.

Consider a 6-dof general parallel manipulator, as shown in figure 2. Each leg has a prismatic actuator. The legs are joined at the ends, to the $F B$ and $M P$, by a universal and a spherical joint, respectively. Let the connection points of the $i^{\text {th }} \operatorname{leg}$ on the $M P$ and $F B$ be denoted by $A_{i}$ and $B_{i}$, respectively. Also, let two coordinate frames $B$ and $M$ be attached to the $F B$ and $M P$ at the points $O$ and $P$, respectively. The position vectors of points $B_{i}$ and $A_{i}$ expressed in frame $B$ are denoted as $b_{i}$ and $p_{i}$, respectively.

The kinematic design problem involves the determination of coordinates of the connection points $A_{i}$ and $B_{i}$ for each leg. Hence, 6 design parameters are associated with each leg. Thus, a 6 legged non-redundant parallel manipulator has 36 design parameters, which are required to be evaluated so that the design criteria is satisfied. But, since the number of these design parameters is quite large and governing equations are nonlinear, their optimal values must be determined numerically. This is achieved by formulating the design problem as a nonlinear constrained optimization problem. The optimization criteria used in the present work is discussed in section 2.3. Prior to that, an elaboration on the earlier design methods will be given in the next section. This will aid comparisons with the method proposed in the present work.

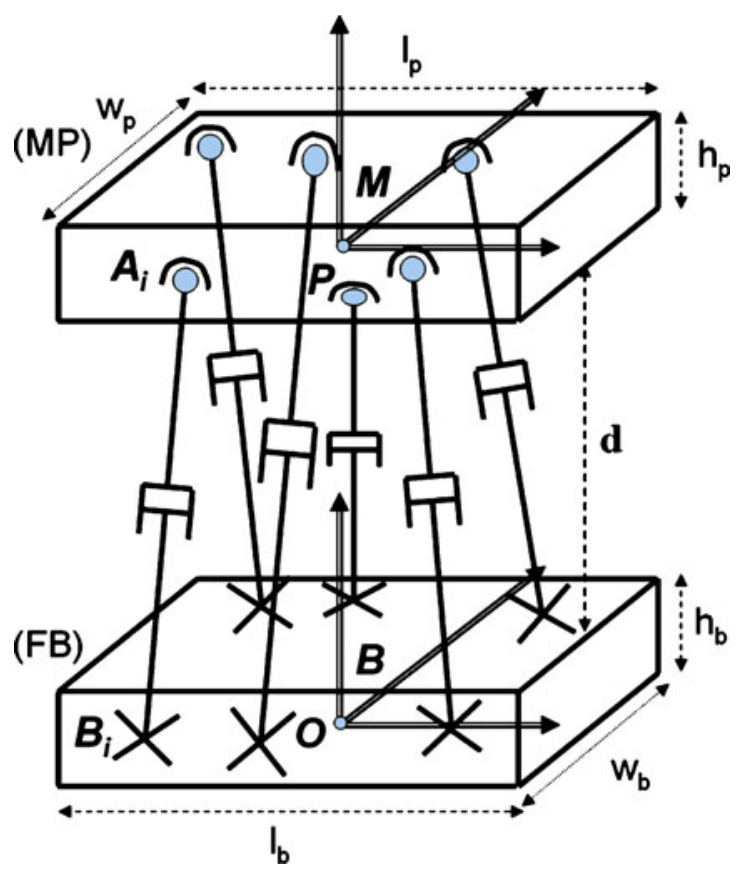

Figure 2. General 6-dof parallel manipulator. 


\subsection{Design method}

As discussed above, the number of design parameters involved is quite large, and so optimizing them simultaneously is computationally expensive. A very common approach to sort out this difficulty is to reduce the number of design parameters. This can be done by putting some constraints on the relative positions of joint centers, which means choosing some standard architecture for the platforms, and then optimizing the remaining parameters. For example, one can assume all the platform connection points to lie on the same plane. As soon as the designer makes this assumption, the number of design parameters reduces to 26 from 36 for a non-redundant 6 dof parallel manipulator.

The most commonly used architecture for the platform is of a semi-regular hexagonal ${ }^{2}$ type. $^{2}$ Design parameters, associated with a parallel manipulator having such an architecture, are only five. Ma \& Angeles (1991) compared this architecture with some of the other architecture on the basis of numerical stability of Jacobian matrix and showed that this architecture is better than the others.

In the present work, the previous approach of reducing the design parameters by choosing an architecture for the platforms is not followed. The connection points are not forced to lie on the same plane on the respective platforms, rather they are given the freedom to be non-coplanar. Hence, in a more general approach all the 36 parameters (for non-redundant 6 dof of parallel manipulator) are optimized simultaneously. Dealing with a complete set of parameters increases the likelihood of getting a better design.

Apart from being optimal, another important aspect related to design procedure is that the obtained design should be feasible from a practical point of view. For example, a design procedure, resulting in an architecture with joint locations such that the two spherical joints almost coincides, is not acceptable as it is not possible to fabricate two spherical joints very close to each other. Thus, while designing, these physical constraints should also be taken into consideration. In the present design procedure, these considerations appear as constraints.

\subsection{Optimization criteria}

The design process is a difficult problem because of the lack of a well-defined objective function which incorporates both kinematic as well as dynamic aspects. If the focus is on kinematics and statics, rather than dynamics, one can define a measure in terms of force transformation matrix ${ }^{3}$ that maps the joint torques to the end effector forces.

Static analysis of the parallel manipulator yields the expression

$$
\Gamma=\mathbf{H F},
$$

where

$\Gamma$ : Generalized external force vector,

F: Actuation force vector at legs to support $\boldsymbol{\Gamma}$,

H: Force transformation matrix.

\footnotetext{
${ }^{2}$ An arrangement of six point on a circle in such a manner that each alternate pair makes an angle of $120^{\circ}$ at the centre.

${ }^{3}$ Equivalently, in terms of the Jacobian.
} 
Force transformation matrix $\mathbf{H}$ maps leg forces to the force and moment at the moving platform. This mapping from the articular forces to the wrench at the moving platform is highly dependent on how well-conditioned is the matrix $\mathbf{H}$. This state of the matrix $\mathbf{H}$ is directly related to its condition number $\mathcal{K}(\mathbf{H})$, which is defined as the ratio of maximum singular value to the minimum singular value, as

$$
\mathcal{K}(\mathbf{H})=\frac{\sigma_{\max }}{\sigma_{\min }} .
$$

Condition number of a matrix measures the round-off error amplification on solving a system of linear algebraic equations associated with that matrix. This linear system of equations arises in both resolved rate control scheme and force-feedback algorithms, hence it is of ample importance to study the state of matrix $\mathbf{H}$. This matrix is configuration dependent, and so the following conditions need to be introduced in the first place.

2.3a Isotropic: This condition corresponds to a configuration at which all the singular values of $\mathbf{H}$ are equal and non-zero, hence condition number $\mathcal{K}(\mathbf{H})$ is 1 . This configuration is termed as the isotropic configuration. In an isotropic configuration, the round-off error amplification on solving a system of linear algebraic equation, is minimum. This configuration is most desirable in the sense of dexterity, because at this configuration the mechanism can withstand external wrench from any random direction equally well.

2.3b Singular: This condition corresponds to a configuration at which one or more singular values of $\mathbf{H}$ are zero, hence condition number $\mathcal{K}(\mathbf{H})$ is infinity. This configuration is termed as singular configuration and is undesirable for general applications, since it corresponds to unconstrained instantaneous motion of the moving platform that cannot be controlled through actuators.

2.3c Ill-conditioned: In certain cases, even when the manipulator is not at a singular configuration but close to it, the force required at some of the legs to counteract the external wrench is enormously high. Mathematically speaking, near the singular configuration, matrix $\mathbf{H}$ is ill-conditioned as one or more singular values are close to zero.

When $\mathbf{H}$ is singular (infinite condition number) the mechanism under analysis is in a singular configuration. This implies that in such configurations, the mechanism cannot support external loads coming from certain directions. Therefore, it is desirable that the manipulator works in the regions which are well removed from the singular and ill-conditioned regions. The aim of the present work is to search for a parallel manipulator whose home configuration is nearly isotropic. In other words, we aim to minimize the condition number, $\mathcal{K}(\mathbf{H})$.

In this context, it is important to point out that some authors tend to consider a scaled version of the matrix $\mathbf{H}$, as its three lower rows are of a different dimension when compared to the three upper ones. Dimensional homogeneity can be obtained by dividing the three lower rows with a characteristic length arising out of the application in which the manipulator is supposed to be used. For a designer targetting a particular domain of application, it is advisable to work out a linear motion which can be considered to be of equivalent magnitude to a unit rotation (one radian) and this can be used to scale matrix $\mathbf{H}$. In the current work, we refrain from using such a scaling for two reasons. First, the focus of the current paper is the method and not any particular application. The algorithmic scheme operates equally with or without scaling, only with quantitative difference in the final result. Secondly, for generic applications, the base/platform dimensions 
quite often turn out to fix the significant length-scale, in which case the transformation $\mathbf{H}$, as it is, happens to be appropriately scaled, since the same base/platform dimensions enter into the three lower rows that is said to introduce the dimensional heterogeneity.

In the event of applying scaling to matrix $\mathbf{H}$, it is important to stress that, the choice of the 'significant length' should be based on a practical engineering consideration. The practice of determining this 'significant length' by imposing the condition of isotropy, as in (Fattah \& Hasan Ghasemi 2002; Fassi et al 2005), lacks conceptual priority, since it is equivalent to choosing the scale factor in a manner simply to confer the property of isotropy to a configuration by definition! For being of any engineering relevance, the scale factor must have a physical and practical basis, and the conditioning of the configuration can be meaningfully discussed only after the scales are decided.

As it is difficult to incorporate two joints very close to each other, the minimum distance between any two joints on the $M P$ and $F B$ are constrained to be above a certain value. Besides, practical requirements put some bounds on the design parameters. For incorporating these bounds, the present work considers two cuboids of certain dimensions separated by a distance ' $d$ ' as shown in figure 2. All the connection points of respective platforms are forced to lie within these two cuboids.

Finally, note that the condition of the manipulator depends on the design as well as the configuration. Use of global indices, e.g., the one suggested by Gosselin \& Angeles (1991) are effective for evaluation of a given manipulator, but not practical for iterative design involving a large number of design variables. Besides, use of such prohibitively costly indices do not seem necessary either, as the present results demonstrate. Rather, we incorporate the effect of configuration in a different manner. We target the determination of the design of the manipulator simultaneously with a so called home configuration, around which the conditioning is good, with the intention to build up the workspace of the manipulator around this optimal configuration. As such, all the coordinates in the following formulation refer to a single frame of reference, i.e., the fixed one.

\subsection{Formulation of optimization problem}

With the various aspects of the design discussed above, the design problem can now be formulated as below.

Minimize

subject to

$$
\begin{aligned}
& \left\|\mathbf{A}_{\mathbf{i}}-\mathbf{A}_{\mathbf{j}}\right\| \geq d_{\min }, \\
& \left\|\mathbf{B}_{\mathbf{i}}-\mathbf{B}_{\mathbf{j}}\right\| \geq d_{\min }, \\
& -l_{p} / 2 \leq \mathbf{A}_{\mathbf{x i}} \leq l_{p} / 2, \\
& -w_{p} / 2 \leq \mathbf{A}_{\mathbf{y i}} \leq w_{p} / 2, \\
& d+h_{b} \leq \mathbf{A}_{\mathbf{z i}} \leq d+h_{p}+h_{b}, \\
& -l_{b} / 2 \leq \mathbf{B}_{\mathbf{x i}} \leq l_{b} / 2, \\
& -w_{b} / 2 \leq \mathbf{B}_{\mathbf{y i}} \leq w_{b} / 2, \\
& 0 \leq \mathbf{B}_{\mathbf{z i}} \leq h_{b}
\end{aligned}
$$

where $i, j: 1,2 \cdots n$.

Here, $l_{p}, w_{p}, h_{p}, l_{b}, w_{b}, h_{b}, d$ and $d_{\min }$ are the input parameters, $d_{\min }$ and $n$ being the minimum distance between the joints and the number of legs, respectively. 


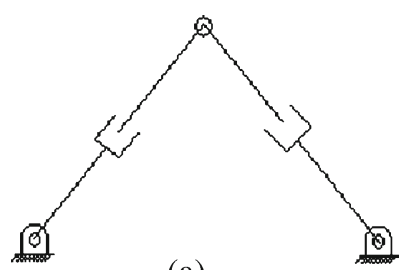

(a)

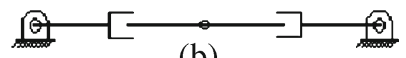

(b)

Figure 3. 2-dof Non-redundant parallel manipulator.

\subsection{Design of a 6-dof non-redundant parallel manipulator}

The problem stated above can be solved for designing a non-redundant 6-dof parallel manipulator with six legs i.e., $n=6$, for certain values of input parameters. In the next section this design optimization problem is solved for two different sets of input parameters.

A set of initial values for the design parameters is required to initiate the numerical process. The initial guess in the present case corresponds to an $\mathrm{SSM}^{4}$ having kinematic parameters which were optimized within the same bounds and constraints for minimum condition number. The choice of the initial guess parameters have been deliberately taken like this in order to establish that there could exist better designs, which the present method expects to find.

\subsection{Design of a 6-dof redundant parallel manipulator}

Introducing redundancy into the system and making it more reliable has long been a useful concept. Redundancy is introduced into the system by giving more number of inputs than the minimal requirement. This concept of introducing redundancy has been utilized in case of parallel manipulators to enhance the usable workspace.

Parallel manipulators suffer from a disadvantage of having less workspace as compared to their serial counterpart and this disadvantage is exacerbated by the presence of singularities within the workspace. As discussed earlier in section 2.3, at singular configurations inside the workspace, a parallel manipulator gains additional degrees of freedom and becomes unstable. This adverse situation can be overcome by introducing an extra leg in parallel to existing ones. This is termed as introducing force redundancy into the parallel manipulator by Dasgupta \& Mruthyunjaya (1998). The effect of adding an extra leg can be viewed as the particular extra input, which will make the workspace of manipulator more usable.

For illustration, consider a 2-dof parallel manipulator shown in figure 3a. It consists of two legs, each having an active prismatic and two passive revolute joints at the ends. Consider a particular configuration of this manipulator as shown in figure $3 \mathrm{~b}$. In this configuration, both the legs are collinear and hence the manipulator can support an external force only along the line of collinearity. Any force having a component perpendicular to the line of collinearity will make the manipulator unstable. This is an example of singular configuration. To get rid of such kind of situations, we can add another leg to the manipulator; but, then, the question arises - how? The importance of this question cannot be overlooked. As shown in figure $4 \mathrm{a}$, the addition of an

\footnotetext{
${ }^{4}$ Parallel manipulator with semi-regular hexagonal platforms.
} 


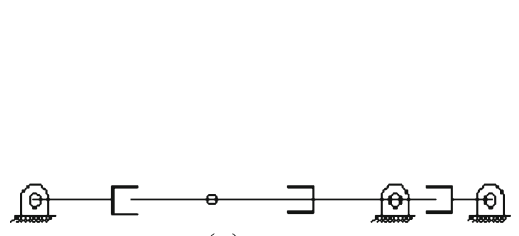

(a)

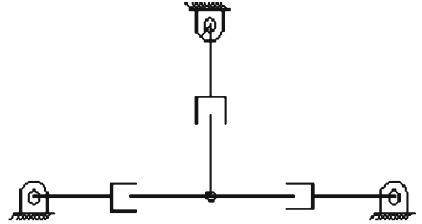

(b)

Figure 4. 2-dof Redundant parallel manipulator.

extra leg does not improve the situation because the manipulator still cannot support the force coming in the direction perpendicular to the line of collinearity. On the other hand, if a leg is added in the manner as shown in figure $4 \mathrm{~b}$, the manipulator can withstand a force coming from any random direction. From this, we can conclude that the addition of an extra leg may or may not remove the singularities present within the workspace. Hence, the way in which redundancy is introduced into the parallel manipulator is of paramount importance.

For a 6-legged non-redundant parallel manipulator, the problem of adding an extra leg and making it redundant, is not as simple as the example discussed above. The extra leg should serve its intended purpose i.e., it should result in the reduction of the dimension of singularity manifold present within the workspace of a non-redundant manipulator. Moreover, it should also be compatible with the design criteria (minimizing $\mathcal{K}(\mathbf{H})$, in the present case).

The extent to which the working region becomes free from singularity also depends on the way in which redundancy is introduced into the system. There can be two ways by which redundancy can be introduced into the parallel manipulator.

Case I: Adding another leg in parallel to the existing six legs of a non-redundant manipulator. Thus, designing the redundant manipulator in this way is a two-step problem. First, a nonredundant manipulator is designed by solving the problem formulated in section 2.4 with $n=6$. Thereafter, the seventh leg is introduced into the previously obtained manipulator by solving the same optimization problem again for $n=1$. The problem is solved for several different initial guesses. The results presented here correspond to those initial guesses which led to the minimum values of the objective function.

Case II: Conceptualizing a redundant manipulator from the very initial stage of kinematic design procedure, with seven legs and solving the optimization problem with $n=7$. The output of the above design (case I) is used here as the initial guess. This exercise conclusively demonstrates the superiority of the current procedure, since any iterative improvement of the design can take place only if the initial guess is not an optimal solution!

Both these ways of introducing redundancy is numerically demonstrated and compared in the next section for two different sets of input parameters.

\section{Results and discussion}

In this section, the formulation is validated for the kinematic design of 6-dof ordinary and redundant parallel manipulators. The numerical optimization problems appearing from the formulation are solved by the usual MATLAB tools, which turn out to be quite effective for the problem and obviate the need for further sophisticated algorithms. Contour plots have been used to show the difference between the condition of workspace before and after the introduction of redundancy. 
All the plots are obtained by moving the $M P$ with respect to $F B$ in the XY plane around the home configuration and keeping the orientation constant, parallel to the base frame.

\subsection{Numerical case-study}

Set (i) $l_{p}=50 \mathrm{~cm}, w_{p}=70 \mathrm{~cm}, h_{p}=25 \mathrm{~cm}, l_{b}=80 \mathrm{~cm}, w_{b}=100 \mathrm{~cm}, h_{b}=30 \mathrm{~cm}, d=30$ $\mathrm{cm}, d_{\min }=10 \mathrm{~cm}$.

\section{- Non-redundant}

Condition number corresponding to initial guess is $\mathcal{K}(\mathbf{H})=14.88$.

After convergence, condition number corresponding to the final configuration is $\mathcal{K}(\mathbf{H})=1.31$.

\section{- Redundant}

\section{- Case I}

Condition number corresponding to initial guess is $\mathcal{K}(\mathbf{H})=3.00$.

After convergence, condition number corresponding to final configuration is $\mathcal{K}(\mathbf{H})=2.11$.

\section{- Case II}

Condition number corresponding to initial guess is $\mathcal{K}(\mathbf{H})=2.11$.

After convergence, condition number corresponding to the final configuration is $\mathcal{K}(\mathbf{H})=1.20$.

Set (ii) $l_{p}=40 \mathrm{~cm}, w_{p}=40 \mathrm{~cm}, h_{p}=20 \mathrm{~cm}, l_{b}=40 \mathrm{~cm}, w_{b}=40 \mathrm{~cm}, h_{b}=20 \mathrm{~cm}, d=$ $30 \mathrm{~cm}, d_{\min }=10 \mathrm{~cm}$.

\section{- Non-redundant}

Condition number corresponding to initial guess is $\mathcal{K}(\mathbf{H})=32.87$.

After convergence, condition number corresponding to the final configuration is $\mathcal{K}(\mathbf{H})=$ 1.80 .

\section{- Redundant}

\section{- Case I}

Condition number corresponding to initial guess is $\mathcal{K}(\mathbf{H})=14.04$.

After convergence, condition number corresponding to final configuration is $\mathcal{K}(\mathbf{H})=1.94$.

\section{- Case II}

Condition number corresponding to initial guess is $\mathcal{K}(\mathbf{H})=1.94$.

After convergence, condition number corresponding to the final configuration is $\mathcal{K}(\mathbf{H})=1.70$.

The kinematic design parameters obtained after the convergence of the numerical algorithm correspond to a manipulator having home configuration very close to isotropic. The architecture obtained for the platforms do not resemble any standard architecture generally used in practice. Also, in comparison to the best SSM, this design is much better.

As explained earlier, each of the contour plots shown in the figures are obtained by positioning the platform-reference-point vertically above (along $z$-direction) the base-reference-point at a certain height and calculating the condition number of the force transformation matrix. There are infinite points on a plane and it is impossible to make calculation at each and every point. 


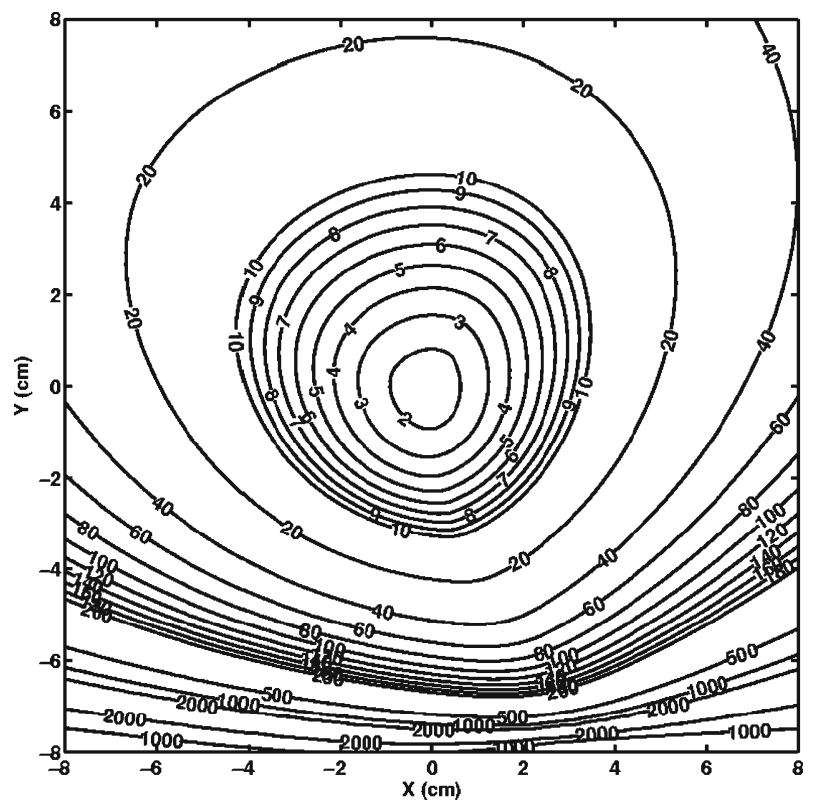

Figure 5. Variation of condition number around the best point at $Z=30 \mathrm{~cm}$ for 6-dof non-redundant manipulator for set (i).

Hence, we discretize the workspace into a grid and then at each grid point calculate the condition number. The condition number at a point signifies the closeness of that point to singularity. In general, the smaller the condition number, the greater the distance from the singular region. In fact, all the singular points lie on one or more hypersurfaces. Similarly, all the points having the same condition number could be considered to be lying on similar hypersurfaces, a projection of which we show in the plots. With the present level of discretization, points having a condition number of more than 2000 have been considered to be singular, in order to capture the contours in
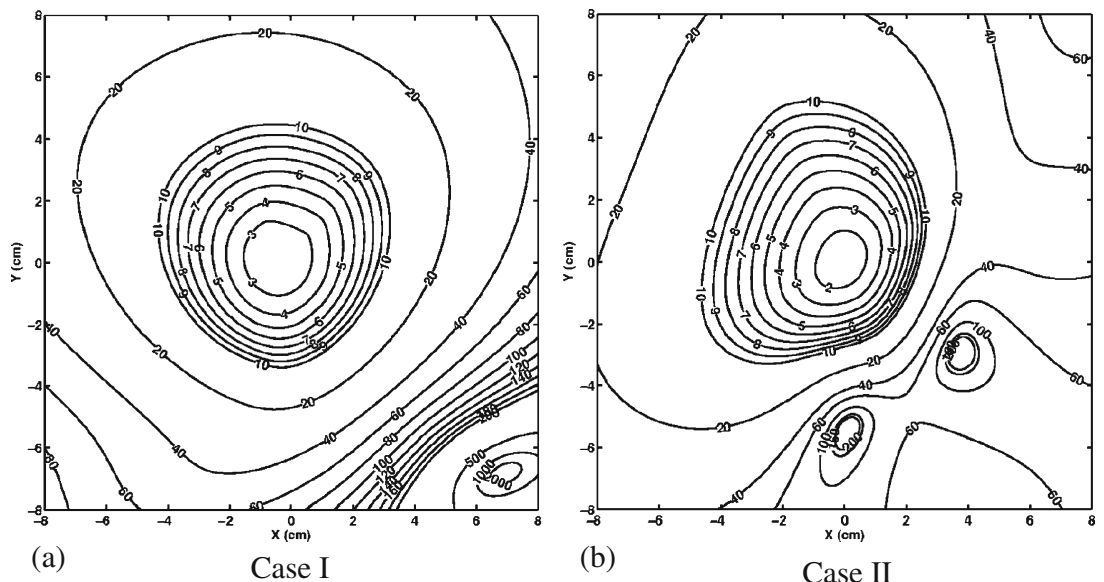

Figure 6. Variation of condition number around the best point at $Z=30 \mathrm{~cm}$ for 6 -dof redundant manipulators for set (i). 


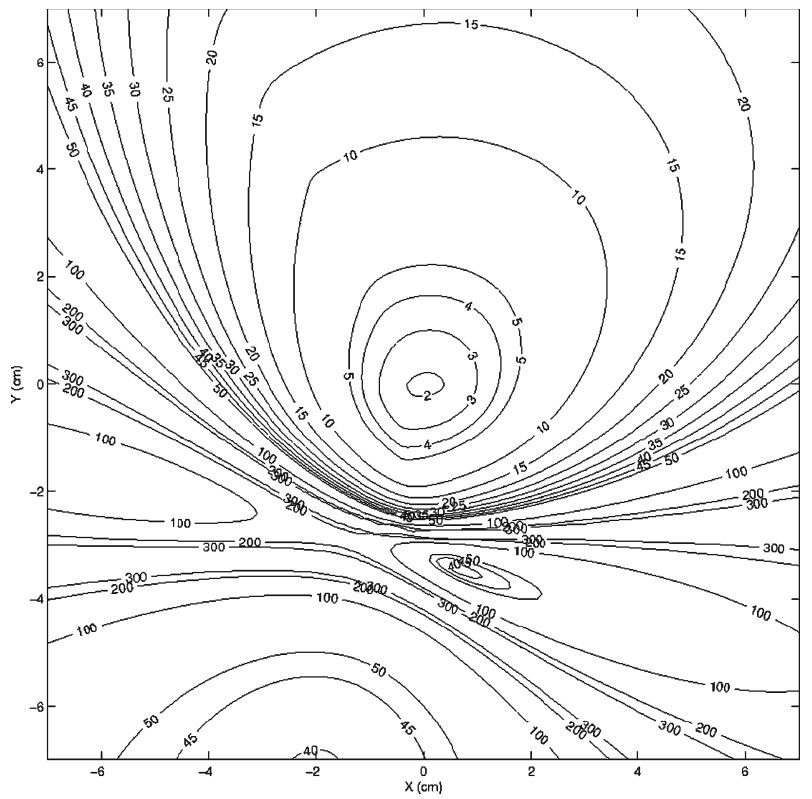

Figure 7. Variation of condition number around the best point at $Z=30 \mathrm{~cm}$ for 6-dof non-redundant manipulator for set (ii).

a continuous manner. With a finer discretization, a larger cut-off value may represent singularity, and vice versa.

The dense regions appearing in figure 5 show the presence of singularity within the workspace, for the first set of input parameters. The condition number corresponding to these regions are as high as 2000. These regions divide the whole workspace into small sub-regions, which are practically usable. Thus, a path for the end-effector from one sub-region to another cannot be

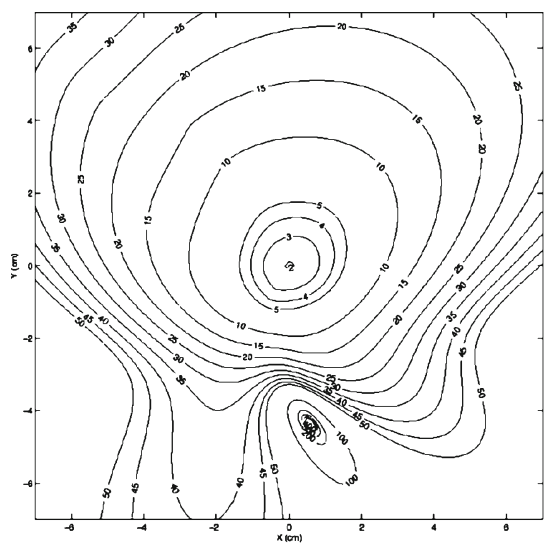

(a)

Case I

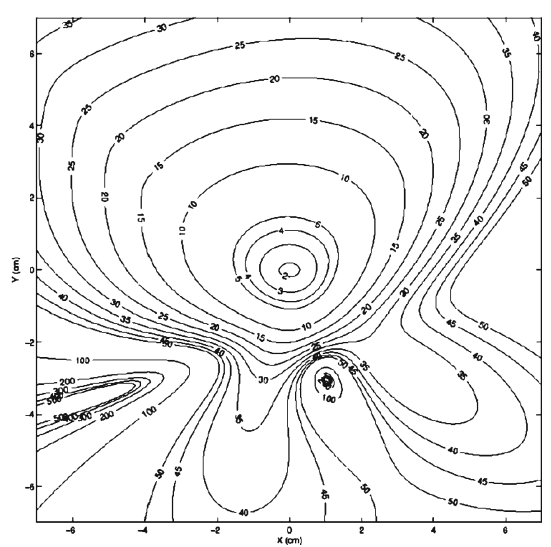

(b)

Case II

Figure 8. Variation of condition number around the best point at $Z=30 \mathrm{~cm}$ for 6 -dof redundant manipulators for set (ii). 


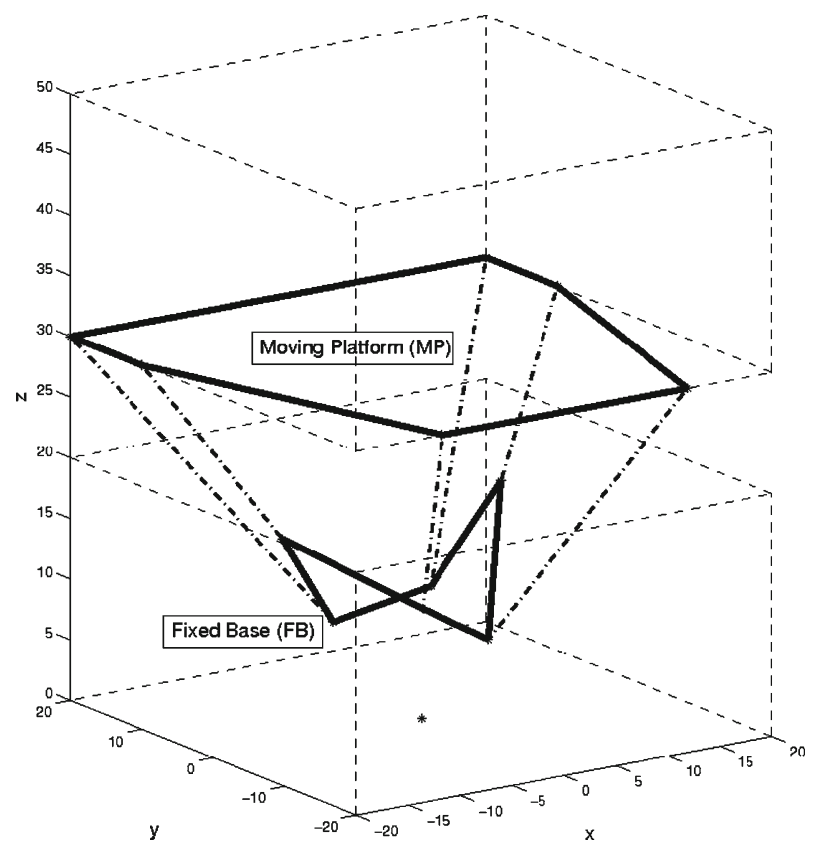

Figure 9. Joint locations obtained for 6-dof non-redundant manipulator for set (ii).

obtained without encountering singular regions. Hence, it is desirable to remove these dense regions from the workspace.

Figure 6 shows the effect of introducing force redundancy. One can clearly see that the singular regions have either collapsed or they have been relocated somewhere near the boundary of the workspace. The severity of the ill-conditioning is also reduced as open ended contours with high condition numbers such as 2000 contract to very small regions (see figure 6b), effectively constituting small holes in the usable workspace but not posing insurmountable barriers in path

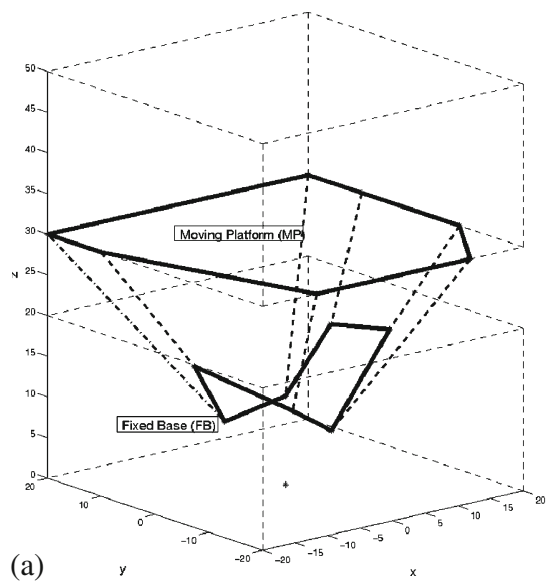

Case I

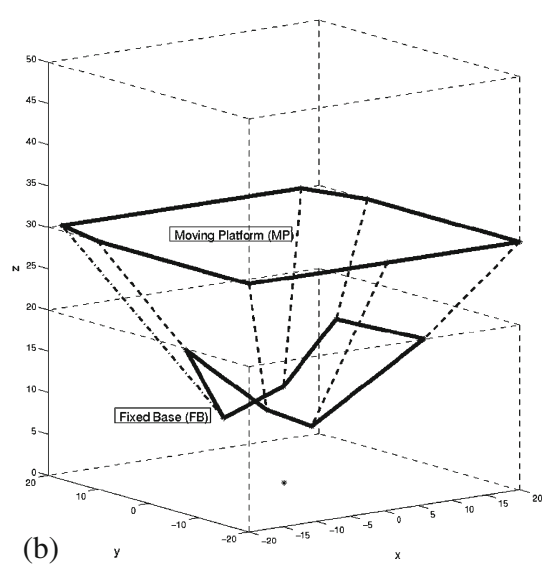

Case II

Figure 10. Joint locations obtained for 6-dof redundant manipulators for set (ii). 


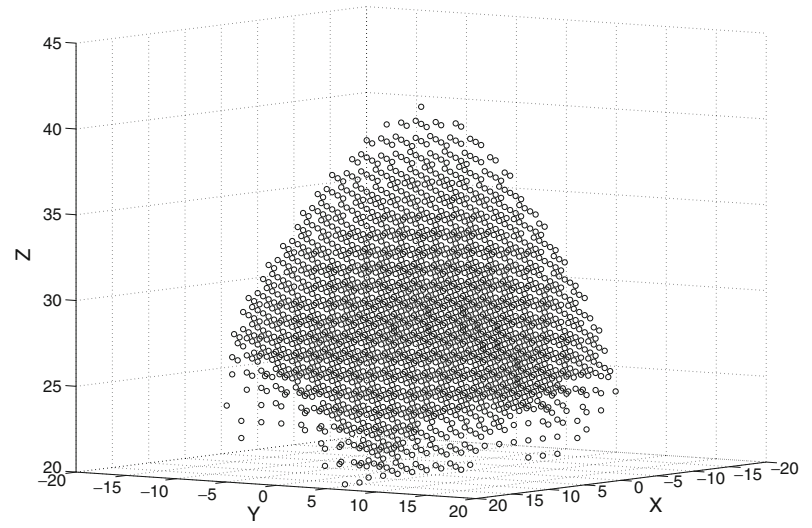

Figure 11. Different locations reached by the non-redundant manipulator within the workspace with a constant orientation for set (ii).

planning. Similarly, for the second set of input parameters, figures 7 and 8 show condition of the workspace for a non-redundant and redundant manipulator, respectively. Also, figures 9 and 10 give a kinematic design of the manipulators obtained. The coordinates of the connection points at the fixed base and moving platform corresponding to kinematic design of the figures $(9,10)$ are given in the appendix I.

Note that the above analysis shows the quality of the workspace at a fixed value of $Z=30$ $\mathrm{cm}$. To examine the 3-dimensional workspace, we can vary $Z$ and obtain the different locations reached by the manipulator. Figures 11 and 12 show the different locations reached by the manipulator within the workspace with a constant orientation. However, these figures do not provide any information regarding the quality of the workspace. Hence, different cross-sections showing the contours of the condition number in parallel planes are obtained by varying $Z$. Figure 13 shows the contours of condition number of the manipulators of set (ii), around the best point $(Z=30 \mathrm{~cm})$, at different cross-sections. It is clearly observed that the value of the condition number is increasing as we move away from the best point.
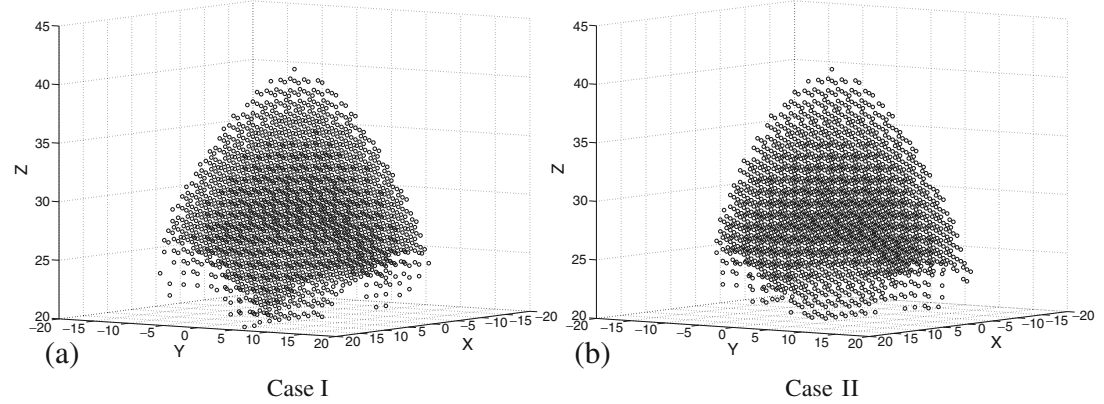

Figure 12. Different locations reached by the redundant manipulator within the workspace with a constant orientation for set (ii). 

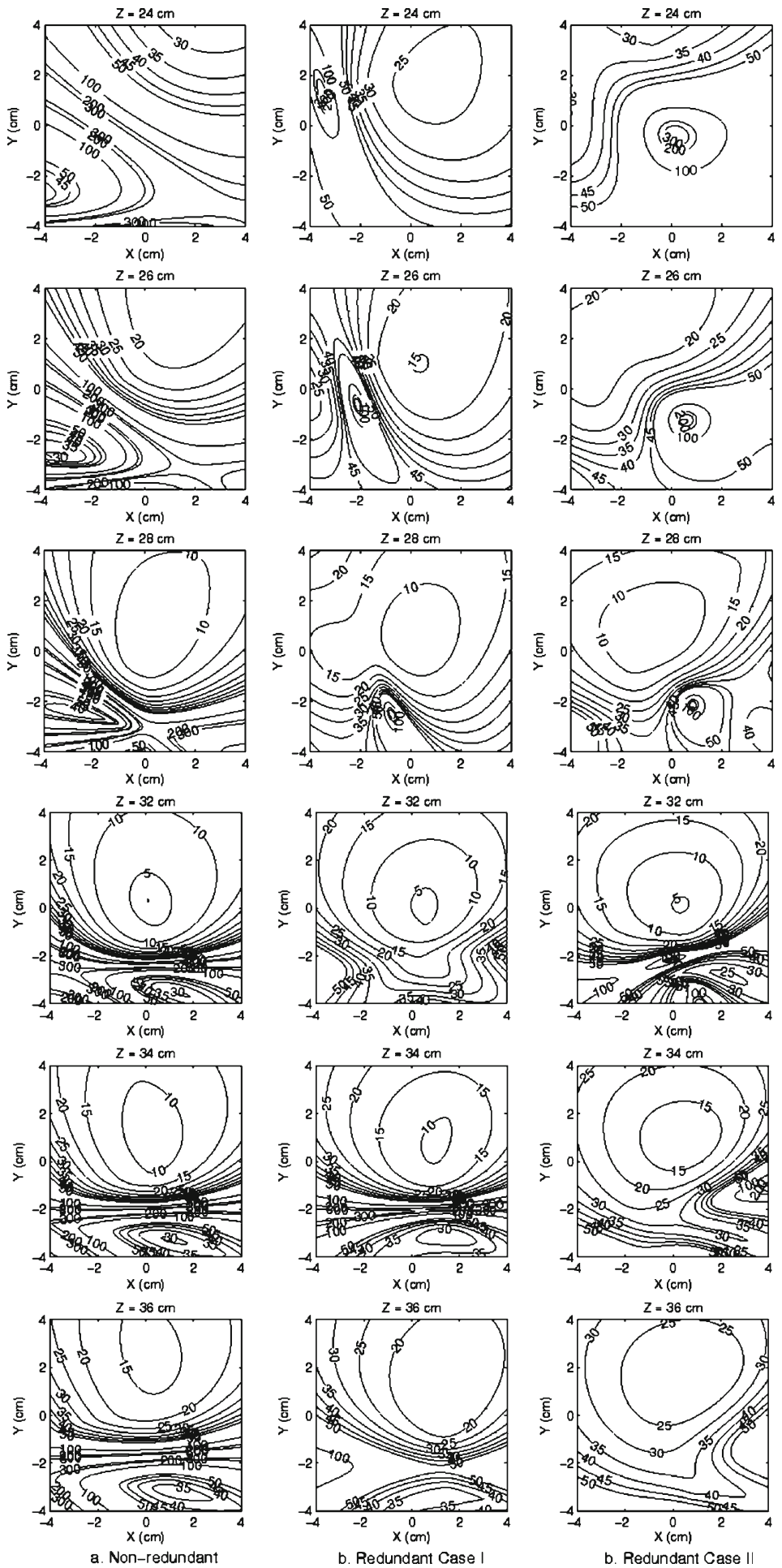

Figure 13. Variation of condition number for different heights of the platform, for the set (ii). 
Difference between the two ways of introducing redundancy can be seen if a comparison is made either with the help of contour plots (figures $6 \mathrm{a}$ and $\mathrm{b}$ ) or by comparing the minimum value of objective function obtained in the two cases. Both the comparisons reveal that introducing redundancy in the very initial phase of the design process results in better design than introducing redundancy to previously existing designs.

From a practical standpoint, introduction of force redundancy has more value than simply to enhance the workspace quality, as considered in the present paper. Related to the current objective, efficient load-sharing with the help of additional leg(s) can be worked out for generic loads (Dasgupta 2002). Besides, such redundancy can be utilized for completely different purposes as well. For example, in an interesting recent work, Yi et al (2005) report designs of redundant parallel manipulators for fault tolerance, which is a quite worthwhile objective, particularly for applications demanding high safety norms. In that case, however, design considerations are quite different, in which conditional optimality of the manipulator in spite of a failed actuator is in focus rather than the combined optimality of all of them!

\section{Conclusions}

In the present work, a general approach for optimal kinematic design of 6-dof parallel manipulators is suggested. In contrast to choosing any specific architecture and reducing the design parameters, a more general approach of considering all the minimally required kinematic design parameters, is followed. In this method, the bounds and constraints are applied in such a manner that the resulting geometry is practically feasible. Besides, the same approach is followed for designing 6-dof redundant manipulator with seven legs. The two ways of introducing redundancy have been analysed and compared. The numerical results demonstrate that it is better to conceptualize a redundant mechanism in its kinematic design phase rather than introducing redundancy in an existing mechanism. Mathematically speaking, for the optimization problem, to obtain a better extremum, it is better to consider all the parameters simultaneously rather than considering them in different phases.

A detailed mechanical design of the manipulator can be made on the basis of kinematic design obtained from the synthesis used in the present work. For the present analysis, static criteria has been used for the designing. In future, some dynamic criteria may also be considered for designing the parallel manipulator.

\section{Appendix I}

Coordinates of the connection points at the fixed base and moving platform for set (ii)

\section{I.1 For non-redundant manipulator}

$$
\text { Fixed Base }=\left[\begin{array}{ccc}
11.07 & 4.95 & 16.60 \\
5.34 & 6.46 & 8.49 \\
-4.56 & 5.73 & 7.31 \\
-10.27 & 4.51 & 15.43 \\
-5.10 & -7.58 & 11.66 \\
3.45 & -4.36 & 6.97
\end{array}\right], \text { Moving Platform }=\left[\begin{array}{ccc}
20.00 & 10.00 & 30.00 \\
20.00 & 20.00 & 30.00 \\
-20.00 & 20.00 & 30.00 \\
-20.0 & 10.00 & 30.00 \\
-11.70 & -20.00 & 30.00 \\
11.88 & -20.00 & 30.00
\end{array}\right]
$$


I.2 For redundant manipulator: case I

Fixed Base $=\left[\begin{array}{ccc}11.07 & 4.95 & 16.60 \\ 5.34 & 6.46 & 8.49 \\ -4.56 & 5.73 & 7.3 \\ -10.27 & 4.517 & 15.43 \\ -5.10 & -7.58 & 11.66 \\ 3.45 & -4.36 & 6.97 \\ 11.96 & -4.90 & 17.99\end{array}\right]$, Moving Platform $=\left[\begin{array}{ccc}20.00 & 10.00 & 30.00 \\ 20.00 & 20.00 & 30.00 \\ -20.00 & 20.00 & 30.00 \\ -20.00 & 10.00 & 30.00 \\ -11.71 & -20.00 & 30.00 \\ 11.88 & -20.00 & 30.00 \\ 20.00 & -8.16 & 30.00\end{array}\right]$

\section{I.3 For redundant manipulator: case II}

Fixed Base $=\left[\begin{array}{ccc}11.75 & 5.49 & 17.37 \\ 5.14 & 7.07 & 9.76 \\ -4.52 & 5.88 & 7.38 \\ -10.74 & 5.05 & 16.54 \\ -8.25 & -7.72 & 11.17 \\ 0.92 & -4.32 & 7.47 \\ 11.81 & -11.97 & 17.94\end{array}\right]$, Moving Platform $=\left[\begin{array}{ccc}20.00 & 10.36 & 30.00 \\ 17.33 & 20.00 & 30.00 \\ -18.02 & 20.00 & 30.00 \\ -20.00 & 10.19 & 30.00 \\ -20.00 & -20.00 & 30.00 \\ 0.39 & -20.00 & 30.00 \\ 19.99 & -20.00 & 30.00\end{array}\right]$.

\section{References}

Chablat D, Wenger P, Angeles J 1998 Iso-conditioning loci of a class of closed-chain manipulators, Proc. IEEE International Conference on Robotics and Automation 3: 1970-1975

Dasgupta B 2002 Redundancy resolution schemes for parallel manipulators with force redundancy. Proceedings of the WORKSHOP on Fundamental Issues and Research Directions for Parallel Mechanisms and Manipulators, Quebec City, Canada, pages 257-260

Dasgupta B, Mruthyunjaya T S 1998 Force redundancy in parallel manipulators: Theoretical and practical issues, Mech. Mach. Theory 33(6): 727-742

Dasgupta B, Mruthyunjaya T S 2000 The Stewart platform manipulator: A review, Mech. Mach. Theory 35(1): 15-40

Dwarakanath T A, Dasgupta B, Mruthyunjaya T S 2001 Design and development of a Stewart platform based force-torque sensor, Mechatronics 11: 783-809

Fassi I, Legnani G, Tosi D 2005 Geometrical conditions for the design of partial or full isotropic hexapods, J. Robot. Syst. 22: 507-518

Fattah A, Hasan Ghasemi A M 2002 Isotropic design of spatial parallel manipulators, Int. J. Robot. Res. 21(9): 811-826

Gosselin C, Angeles J 1988 The optimum kinematic design of planer 3 dof parallel manipulators, J. Mech. Transm. Automn. Des. 110: 36-41

Gosselin C, Angeles J 1990 Singularity analysis of closed-loop kinematic chains, IEEE Trans. Robot. Automn. 6: 281-290

Gosselin C, Angeles J 1991 A global performance index for the kinematic optimization of robotic manipulators, J. Mech. Des. 113: 220-226

Hao F, Merlet J-P 2005 Multi-criteria optimal design of parallel manipulators based om interval analysis, Mech. Mach. Theory 40: 157-151

Hay A M, Snyman J A 2003 Methodologies for the optimal design of parallel manipulators, Int. J. Numer. Methods Eng. 59(1): 131-152 
Huang T, Li M, Li Z X, Chetwyand D G 2004 Optimal kinematic design of 2-DOF parallel manipulators with well-shaped workspace bounded by a specified conditioning index, IEEE Trans. Robot. Automn. 20(3): 538-543

Jafari F, McInroy J E 2003 Orthogonal Gough-Stewart platforms for micromanipulation, IEEE Trans. Robot. Automn. 19(4): 595-603

Klein C A, Blaho B E 1987 Dexterity measures for the design and control of kinematically redundant manipulators, Int. J. Robot. Res. 6(2): 72-83

Liu X-J, Wang J, Pritschow G 2005 A new family of Spatial 3-DOF fully parallel manipulators with high rotational capability, Mech. Mach. Theory 40: 475-494

Ma O, Angeles J 1990 The concept of dynamic isotropy and its application to inverse kinematics and trajectory planning, Proc. IEEE International Conference of Robotics and Automation 1: 481-486

Ma O, Angeles J 1991 Optimum architecture design of platform manipulators, IEEE International Conference of Robotics and Automation 2: 1130-1135

Ma O, Angeles J 1993 Optimum design of manipulators under dynamic isotropy condition, IEEE International Conference of Robotics and Automation 1: 470-475

Merlet J-P 2000 Parallel robots. (Boston, London: Kluwer Academic Publishers)

Merlet J-P 2006 Jacobian, manipulability, condition number, and accuracy of parallel robots, J. Mech. Des. 128(1): 199-206

Merlet J-P, Daney D 2005 Dimensional synthesis of parallel robots with a guaranteed given accuracy over a specific workspace. Proceedings of the 2005 IEEE International Conference on Robotics and Automation Barcelona, Spain, April 2005, pages 942-947

Miller K 2002 Maximization of workspace volume of 3-DOF spatial parallel manipulators, J. Mech. Des. 124: $347-350$

Salcudean S E, Drexel P A, Ben-Dov D, Taylor A J, Lawrence P D 1994 A six degree-of-freedom, hydraulic, one person motion simulator, IEEE International Conference of Robotics and Automation 3: $2437-2443$

Salisbury J K, Craig J J 1982 Articulated hand: Force control and kinematic issues, Int. J. Robot. Res. 1(1): 4-17

Stewart D 1965 A platform with six degrees of freedom, Proc. Inst. Mech. Eng. 180(17): 371-386

Stock M, Miller K 2003 Optimal kinematic design of spatial parallel manipulators: Application to linear delta robot, J. Mech. Des.125(2): 292-301

Wang J, Gosselin C M 2004 Kinematic analysis and design of kinematically redundant parallel mechanisms, J. Mech. Des. 126: 109-118

Yi Y, Jafari F, McInroy J E 2005 Generating class of locally orthogonal Gough-Stewart platforms, IEEE Transaction on Robotics 21(5): 812-820

Zanganeh K E, Angeles J 1997 Kinematic isotropy and the optimum design of parallel manipulators, Int. J. Rob. Res. 16(2): 185-197 